\title{
Cytology of Nilaparvata bakeri (Muir), a Grass-Infesting Planthopper (Homoptera: Delphacidae)
}

\author{
A. A. Barrion ${ }^{1}$ and R. C. Saxena ${ }^{2}$ \\ Department of Entomology, International Rice Research Institute, \\ P. O. Box 933, Manila, Philippines
}

Accepted April 2, 1984

The genus Nilaparvata Distant comprises 14 determined and 2 undetermined species which have been recorded from tropical and temperate zones throughout the world (Okada 1977, Mochida and Okada 1979). About one-half of these are known in Asia and the Pacific region and the remaining in Africa and Central America. Two of these species-N. lugens (Stål) and N. bakeri (Muir), are particularly widely distributed and also occur in the Philippines. The former, commonly known as the brown planthopper, is a pest of rice whereas the latter is mainly a grass feeder. The two species can easily be distinguished from each other on the basis of genitalia.

While the cytology of $N$. lugens has been studied by several workers (Saitoh et al. 1970, Liquido 1978, Den Hollander 1982, Saxena and Barrion 1982a, b, 1983), there is barely any information on this aspect of other Nilaparvata species. We, therefore, investigated the cytology of $N$. bakeri which was found thriving on Leersia hexandra (Swartz) weed grass that grew abundantly in irrigation canals along rice fields at the experimental farm of the International Rice Research Institute (IRRI) in the Philippines.

\section{Material and methods}

Wild populations of $N$. bakeri were collected from $L$. hexandra in irrigation canals adjacent to rice fields on the IRRI farm. They were then mass-reared on potted $L$. hexandra cuttings kept in standard rearing cages in an insectary. Newlyemerged brachypterous males were collected from this stock culture and squashes of their testes were prepared for chromosome studies as described earlier by Saxena and Barrion (1982a). Testicular cells and their chromosomes were observed with a Bausch and Lomb research microscope. Measurements of nuclei and chromosomes were made using the oil immersion objective of the microscope equipped with a linear, graduated ocular micrometer. Calibrated micrometer units were then converted into microns $(\mu)$. Photomicrographs were taken directly from temporary slide preparations.

1 Research Fellow, International Rice Research Institute (IRRI).

2 Principal Research Scientist, International Centre of Insect Physiology and Ecology (ICIPE), P. O. Box 30772, Nairobi, Kenya, based at IRRJ. 


\section{Results}

\section{Chromosome count}

The male genomic complement of $N$. bakeri was found to be $2 n=29$, specifically consisting of 14 homomorphic bivalent autosomes and a univalent X-chromosome. Preliminary investigations of the female gonads revealed a diploid complement of $2 n=30$, consisting of 14 homologous autosomes and a bivalent (XX) sex chromosome.

\section{Meiotic study}

Throughout the course of meiosis, no chromosome with localized kinetochore was detected. As attested by the orientation, broad spindle attachment, and parallel disjunction during anaphase I, $N$. bakeri chromosomes had diffused centromeres.

The first and second meiotic divisions were reductional and equational, respectively. The following qualitative and quantitative results regarding the interphase and the meiotic (M)-phase of the reproductive cell cycle of $N$. bakeri were obtained.

Prior to M-phase was interphase (Fig. 1) wherein testicular cells of $N$. bakeri were observed to be oval and measured $45 \mu$ and $32 \mu$ in length and width, respectively. Centrally located within each cell was the nucleus having a $12 \mu$ diameter. A tight knot of chromatin threads called the synizetic knot was closely associated with the nucleolus and situated on one side of the nuclear cavity.

At meiosis I, the knot began to loosen and spireme threads came out of the knot. During leptotene stage (Fig. 2), the meiocytes, 13-25 $\mu$ long, 10-17.4 $\mu$ wide, possessed nuclei with a tangled maze of chromatin threads associated with the nuclear membrane. Each of the threads was composed of two strands tightly twisted around each other. The nucleus measured $10 \mu$ in length and $8 \mu$ in width. From then on, the nucleus increased in volume and the whole interior cavity became occupied by reticulate threads stretching across the cavity.

Pachytene (Fig. 3) was a rarely encountered stage where the chromosomes appeared as elongated, intertwined, paired threads thicker than those of the preceding stages but were still considerably tenuous and sinuous. They displayed banded appearance of positively heteropycnotic chromomeres and knobs as well as euchromatic gaps at various sites of the chromosome strands. Careful examination revealed that $N$. bakeri had 14 heterochromatic pachytene threads. Only one nucleolus per nucleus was seen. The nucleolus organizing chromosome was identified by its association with the nucleolus. As yet no signs of chiasma formation were to be seen in these loops. Relative chromatin length of the 14 haploid autosomes revealed that the shortest chromosome was .0355 whereas the longest chromosome having the nucleolus organizing region measured .1434 .

At prediplotene or diffuse stage, the autosomes lost their affinity for nuclear stain but the sex chromosome remained heterochromatic. The diplotene nuclei measured $31-45 \mu$ in length and $20-21 \mu$ in width. The lightly stained autosomes were scattered all over the whole cell. The separation of homologs was initiated and chromosomes continued to contract. The positively heteropycnotic X-body was $2.2 \mu$ long and $1.5 \mu$ wide. At later stage of diplotene (Fig. 4), the heterochromaticity of autosomes increased and rendered irregular outlines while the univalent 
$\mathrm{X}$ showed smooth outline and uniform heterochromaticity. The autosomal lengths (in $\mu$ ) were: $3.0,3.0,3.0,3.0,3.0,3.0,3.5,3.5,4.0,4.0,4.0,4.0,5.0$ and 5.0.

During diakinesis, the bivalents or gemini attained clear contours. The chiasmata had been fully symmetrized and most of them had been totally terminalized. Chromosomes reached maximum contraction and thickening. The two univalent chromosomes were associated with each other by chiasmatic connections only at

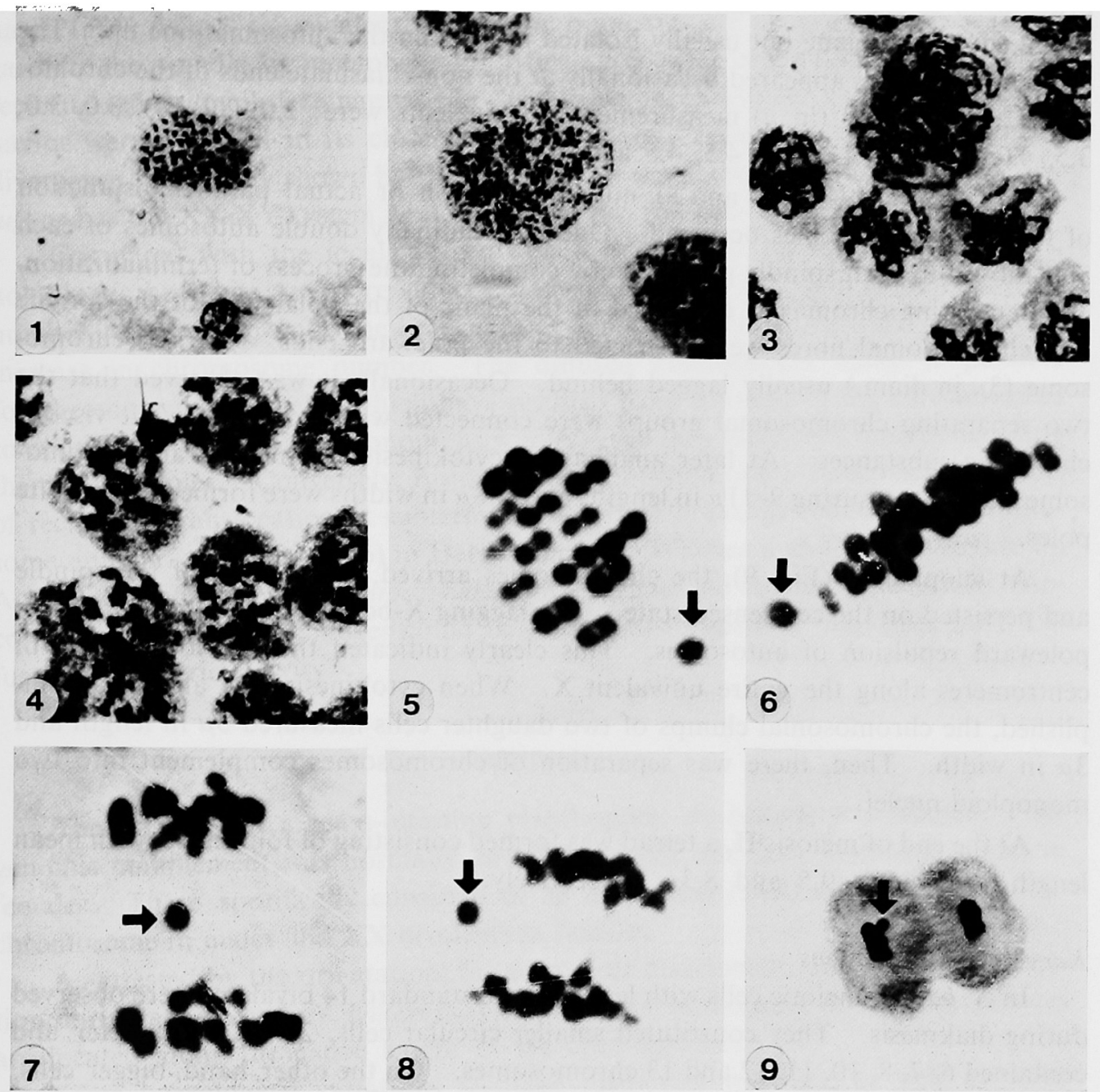

Figs. 1-9. First meiotic stages in spermatocytes of $N$. bakeri. 1, interphase. 2, leptotene. 3, pachytene. 4, diplotene. 5, late diakinesis or prometaphase I. 6, metaphase I. 7 and 8, anaphase I. 9, telophase I. The sex chromosomes are indicated by arrows.

points of twisting or nodes. At the outset of diakinesis, the synapsed homologs became well spaced, often near the nuclear membrane. At later diakinesis or prometaphase I (Fig. 5), homomorphic chromosomes started the movement towards the center forming an elongated cluster. The length (in $\mu$ ) measurements of 14 synapsed homologs were as follows: $2.1,2.4,2.8,3.0,3.0,3.1,3.4,3.7,4.0,4.0,4.1$, 4.4, 4.8 and 4.8. They were closely gradated in size with the shortest element 
approximating one half the length of the longest.

The arrival of synapsed homomorphic gemini at the equator of the spindle began metaphase I (Fig. 6). The bivalents were strongly contracted and densely pressed together in a firm spindle. As a result of vigorous growth during the diffuse stage, the cells and the spindles were quite large providing ample room for the bivalents to orientate in the spindle as dumb-bells. All the bivalents showed axial orientation to the spindle axis. The univalent sex chromosome was also oriented in the equatorial plane but usually isolated itself from the autosomal line up. The chromosome fibres appeared occasionally at the non-chiasmate ends of the chromosomes. The length (in $\mu$ ) measurements of bivalents were: 2.0, 2.0, 3.0, 3.0, 3.0, $3.5,4.0,4.0,4.0,4.0,4.2,4.5,4.5$ and 4.5 .

In anaphase I (Figs. 7 and 8), mutual repulsion or actual parallel disjunction of the two chromosomes occurred. One longitudinally double autosomes of each pair moved to each spindle pole, thereby completing the process of terminalization. The separating chromatids elongated in the plane of the polar axis of the spindle and chromosomal fibres were restricted to the poleward ends. The sex chromosome ( $3 \mu$ in diam.) usually lagged behind. Occasionally,it was observed that the two separating chromosomal groups were connected with thin, somewhat viscous chromatic substances. At later anaphase I, cytokinesis commenced and chromosome clumps measuring $9-11 \mu$ in lengths and $7-8 \mu$ in widths were formed at opposite poles.

At telophase I (Fig. 9), the chromosomes arrived at the poles of the spindle and persisted on the condensed state. The lagging X-body ably caught up with the poleward repulsion of autosomes. This clearly indicated the non-localization of centromeres along the entire univalent $X$. When cytokinesis was almost accomplished, the chromosomal clumps of two daughter cells measured $5 \mu$ in length and $3 \mu$ in width. Then, there was separation of chromosomes complement into two monoploid nuclei.

At the end of meiosis II, a tetrad was formed consisting of four nuclei with mean length and width, 9.5 and $8.3 \mu$, respectively.

\section{Karyological variations}

In $N$. bakeri, meiotic cells with less than the standard 14 bivalents were observed during diakinesis. They constituted smaller circular cells, $27.5 \mu$ in diameter and contained $6,7,8,10,11,12$ and 13 chromosomes. On the other hand, bigger cells, $47 \mu$ in length and $45 \mu$ in width contained increased autosomes of 16, 17, 19 and 20.

\section{Discussion}

Of the 14 species of Delphacidae studied previously (Bhattacharya and Manna 1973, Halkka 1959, 1962, Halkka and Heinonen 1964), chromosome number ranged from 24 to 37 with a peak at 29. N. bakeri is an additional record to the listing of those with 29 chromosomes in males but 30 chromosomes in females. The differential diploid genome in the two sexes of both morphs is attributable to their sex determining mechanism, XX-X0. The male planthopper, therefore, is hetero- 
gametic or capable of yielding two kinds of spermatozoa, $14 \mathrm{I}+\mathrm{X}$ and $14 \mathrm{I}+0$, whereas the female is homogametic and can produce only one type of ovum, $14 \mathrm{I}+\mathrm{X}$.

Insects with holokinetic chromosomes vary in terms of chromosome behavior during meiosis. For instance, in aphids and coccids (Ris 1942, Hughes-Schrader 1955), the bivalent chromosomes were equatorially oriented with regard to the spindle at metaphase I and separate equationally at anaphase I. This type of orientation has been termed "equatorial orientation" by White (1973). On the other hand, in $N$. bakeri, the bivalent holocentric homologs are oriented axially with regard to the spindle at metaphase I. After such axial orientation, the bivalents separate reductionally at anaphase I. The same chromosome orientation and behavior were observed in its closely related species, $N$. lugens. However, genetic divergence was clearly shown by their differential sex determining mechanisms. $N$. lugens has the XX-XY system while $N$. bakeri has the $\mathrm{XX}-\mathrm{X} 0$ system.

Compared with the localized centromere or even polycentric chromosomes, holokinetic bivalents are more subjected to decreases or increases in chromosome number. Simple chromosome breakages or agmatoploidy can provide a basis for increase in chromosome number. The diffused kinetochores make it possible for chromosome fragments which have resulted from splitting of the chromosomes to divide and function as autonomous wholes since they have centromeres directing them to the poles. Conversely, fusion of major portion of chromosomes by means of reciprocal translocation at subterminal points of breakage reduces the chromosome number. Manna (1956) in Heteroptera and Whitten and Taylor (1969) in an Australian leafhopper, Alodeltocephalus draba, envisaged similar mechanisms. According to them, such changes could be easier since insects of this order have diffused centromeric activity.

\section{Summary}

The cytology of a grass-infesting planthopper, $N$. bakeri, was studied. The genomic complement was normally $2 n=29$ in males and $2 n=30$ chromosomes in females. These specifically consisted of 28 autosomes (14II) and a univalent Xchromosome in males and XX bivalents in females.

As attested by the orientation, broad spindle attachment, and parallel disjunction during anaphase $\mathrm{I}, N$. bakeri chromosomes are holocentric or the kinetochores are located along the entire length of the chromosomes.

Some karyological variations included meiocytes with less as well as more chromosomes than the normal genomic constitution. Due to these atypical karyotypes, it can be suggested that $N$. bakeri is chromosomally polymorphic with ample genetic versatility.

\section{References}

Bhattacharya, A. K. and Manna, G. K. 1973. Gonial chromosomes, their behavior and metrical data in nine species of Fulgorids (Homoptera). Cytologia 38: 281-290.

Den Hollander, J. 1982. The chromosomes of Nilaparvata lugens Stål. and some other Auchenorrhyncha. Cytologia 47: 227-236. 
Halkka, O. 1959. Chromosome studies on the Hemiptera, Homoptera, Auchenorryncha. Ann. Acad. Sci. Fen., Series A. IV Biological 45: 1-72.

- 1962. The chromosomes of Membracidae. Hereditas 48: 215-219.

- and Heinonen, L. 1966. Chromosome numbers in 20 American or Sudanese leaf hoppers, with notes on the role of cytology in Homopteran taxonomy. Ann. Ent. Fenn. 32: 11-19.

Hughes-Schrader, S. 1955. The chromosomes of the giant scale Aspidoproctus maximus Louns (Coccoidea-Margaroididae) with special reference to asynapsis and sperm formation. Chromosoma 7: 420-438.

Liquido, N. J. 1978. Morphology, cytology and breeding behavior of Nilaparvata lugens (Stål) (Delphacidae, Homoptera). M. Sci. Thesis, University of the Philippines at Los Baños, Laguna, Philippines.

Manna, G. K. 1956. Cytology and inter-relationships between various groups of Heteroptera. Proc. X Intern. Entomol. Congr. Canada 2: 919-934.

Mochida, O., Tatang Suryana, Hendarsih, and Ayuk Wahyu. 1978. Identification, biology, occurrence and appearance of the brown planthopper. pp. 1-39. In The Brown Planthopper (Nilaparvata lugens Stål). Indon. Inst. Sci., Jakarta: 133 pp.

Ris, H. 1942. A cytological and experimental analysis of the meiotic behavior of the univalent $X$ chromosome in the bearberry aphid Tamalia (=Phyllaphis) coweni (Ckll.). J. Exp. Zool. 90: $267-326$.

Saitoh, K., Kudoh, K. and Mochida, O. 1970. A study of the male meiotic chromosomes in two Homopteran species of economic importance. Sci. Rep. Hirosaki Univ. 17: 31-37.

Saxena, R. C. and Barrion, A. A. 1982a. The technique for chromosome studies of brown planthopper, Nilaparvata lugens (Stål). IRRN 7 (2): 8-9.

- 1982b. Cytogenetic variations in brown planthopper biotypes 1 and 2. IRRN 7 (3): 5.

- 1983. Cytological variations among brown planthopper biotypes 1, 2, and 3. IRRN 8 (2): 14-15.

White, M. J. D. 1973. Animal Cytology and Evolution. Third ed. Cambridge Univ. Press, London. Whitten, M. J. and Taylor, W. C. 1969. Chromosomal polymorphism in an Australian leafhopper (Homoptera, Cicadellidae). Chromosoma (Berl.) 26 (1): 1-6. 